\section{Higher cough flow is associated with lower risk of pneumonia in acute stroke}

\author{
There is little available evidence to \\ demonstrate how cough strength mediates the \\ risk of aspiration-related pneumonia in acute \\ stroke. Our secondary analysis of trial data \\ indicates that risk of pneumonia reduces with \\ increasing peak cough flow (PCF) of voluntary \\ cough (OR 0.994 for each $1 \mathrm{~L} / \mathrm{min}$ increase in \\ PCF, 95\% Cl 0.988 to $1.0, p=0.035)$; and to \\ a lesser degree with increasing PCF of reflex \\ cough (OR 0.998 for each $1 \mathrm{~L} / \mathrm{min}$ increase in \\ PCF, 95\% Cl 0.992 to $1.004, p=0.475$ ). \\ These data serve hypothesis generation. \\ Further studies are needed to confirm these \\ findings and validate their clinical utility. \\ Clinical trial registration number \\ ISRCTN40298220 (post-results).
}

\section{INTRODUCTION}

Cough is the most immediate defence mechanism against aspiration. ${ }^{1}$ It is a commonly encountered clinical belief that strong cough offers some protection from aspiration-related pneumonia, although there is little evidence available to support this. Data from our completed trial of respiratory muscle training in acute stroke (ISRCTN40298220) allowed us to examine the association between cough flow and pneumonia risk. We have previously shown that stroke leads to impairment of both voluntary and reflex cough. ${ }^{2} 3$ Here, we present an exploratory secondary analysis of trial data, examining whether higher peak cough flow (PCF) (indicating stronger cough) might be protective against pneumonia in patients with stroke and swallowing problems.

\section{METHODS}

Data from 72 patients were available for this analysis. Study procedures have been detailed previously. ${ }^{4}$ Briefly, we recruited adults within 2 weeks of stroke, and excluded patients with significant cardiac/ pulmonary disease; neurological conditions other than stroke; orthopaedic conditions affecting respiratory mechanics; inability to cooperate or signs of pneumonia at enrolment. Swallowing function was described according to standardised bedside swallow assessment. $^{5}$ We measured cough flow of volitional and capsaicin-induced reflex cough, using a calibrated pneumotachograph with full face mask. ${ }^{4}$ Pneumonia was observed for 4 weeks following baseline assessment and determined from documented medical diagnosis.

Our analysis was hypothesis-driven, assuming the data structure of a longitudinal observational study and examining only the predictor PCF for outcome pneumonia. First, we stratified the sample according to aspiration risk and pneumonia, and conducted group comparison tests. Second, we used logistic regression to examine the association between PCF and outcome pneumonia in the unsafe-swallow group. Third, we categorised patients in the unsafe-swallow group in two groups of high and low voluntary PCF, using an arbitrary threshold of $400 \mathrm{~L} / \mathrm{min}$; and we calculated the OR for outcome pneumonia according to dichotomised PCF. All analyses were conducted using Stata V.11.2 statistical software.

\section{RESULTS}

Analysis of the sample stratified by aspiration risk showed that PCF of voluntary cough was significantly lower in patients who had unsafe swallow and who developed pneumonia (table 1, see online supplementary figures S1 and S2). Full sample characteristics at baseline are given in online supplementary table S2.

Logistic regression showed a statistically significant association between PCF of voluntary cough and pneumonia (OR 0.994 for each $1 \mathrm{~L} / \mathrm{min}$ increase in PCF, $95 \%$ CI 0.988 to $1.0, \mathrm{p}=0.035)$. The association between PCF of reflex cough and pneumonia was smaller and not statistically significant (OR 0.998 for each $1 \mathrm{~L} / \mathrm{min}$ increase in PCF, 95\% CI 0.992 to $1.004, \mathrm{p}=0.475)$. Goodness of fit indicators were adequate (Pearson $\chi^{2}$ and
Hosmer-Lemeshow tests, $\mathrm{p}>0.05)$. Stata outputs for the logistic regression are given in online supplementary tables S3 and S4.

Categorising patients with unsafe swallow according to a threshold of $400 \mathrm{~L} / \mathrm{min}$ voluntary PCF resulted in 22 patients in the low-PCF category, out of which nine developed pneumonia; and 11 patients in the high-PCF group, out of which two developed pneumonia. The risk of pneumonia was approximately three times higher for patients in the low-PCF group, although this was not statistically significant (OR 3.12, 95\% CI 0.45 to 35.24$)$. The Stata output is given in online supplementary table S5.

\section{DISCUSSION}

Our data lend support to the notion that strong cough protects from aspirationrelated pneumonia. This association was stronger for voluntary cough, which leads us to hypothesise that PCF of voluntary cough might serve as a useful predictor of pneumonia risk in acute stroke. Logistic regression showed that each increase in voluntary PCF by $1 \mathrm{~L} / \mathrm{min}$ reduced the risk of pneumonia by $0.6 \%$ (OR 0.994 ). The equivalent ORs for an increase in voluntary PCF by 50 and $100 \mathrm{~L} / \mathrm{min}$ are approximately 0.73 and 0.53 , respectively.

To illustrate how application of a PCF threshold might inform pneumonia risk in clinical practice, we applied an informed, although somewhat arbitrary cut-off of $400 \mathrm{~L} / \mathrm{min}$ to categorise patients into those with stronger and those with weaker voluntary cough. The appropriate cut-off for PCF in stroke patients is currently not known. The small sample size is a limitation to this analysis. Although we maximised statistical precision by examining only one association of interest, which was defined a priori, studies with larger sample sizes are required to develop more sophisticated multivariable predictor models, which would also allow adjustment for other known risk factors of poststroke pneumonia. $^{6}$

Further limitations to this analysis are trial eligibility criteria, which may have

Table 1 Peak cough flow (PCF) according to 4-week incidence of pneumonia in patients with low aspiration risk (safe swallow) and high aspiration risk (unsafe swallow)

\begin{tabular}{|c|c|c|c|c|c|c|}
\hline & \multicolumn{3}{|c|}{ Low aspiration risk (safe swallow) } & \multicolumn{3}{|c|}{ High aspiration risk (unsafe swallow) } \\
\hline & No pneumonia $(n=37)$ & Pneumonia $(n=2)$ & p Value* & No pneumonia $(n=22)$ & Pneumonia $(n=11)$ & p Value* \\
\hline PCF of voluntary cough (L/min) & $535(264)$ & $546(307)$ & 0.917 & 448 (244) & $252(130)$ & 0.0053 \\
\hline PCF of reflex cough (L/min) & $301(110)$ & $324(168)$ & 0.945 & $276(124)$ & $231(100)$ & 0.277 \\
\hline
\end{tabular}


introduced selection bias not present in observational studies on consecutive patients. Respiratory muscle training in the intervention group may have affected the incidence of pneumonia, but this is unlikely as the trial showed no effect of these exercises on PCF compared with control patients. Although criteria based, pneumonia was physician diagnosed, but detection bias is unlikely as physicians were masked to allocation and to baseline assessments. Any future study of PCF and pneumonia risk would benefit from robust methods for diagnosing pneumonia. ${ }^{7}$ In particular, the potential for diagnosis to be influenced by the diagnosing physician's subjective assessment of cough strength needs to be considered.

Despite limitations, the present analysis provides potentially valuable findings in a little researched field. Measurement of cough flow may provide an objective, device-based method to inform pneumonia risk in patients with stroke and unsafe swallow at the bedside. Further studies are needed to confirm these results and validate their clinical application.

\section{Stefan T Kulnik, ${ }^{1}$ Surinder S Birring, ${ }^{2}$ John Hodsoll, ${ }^{3}$ John Moxham, ${ }^{2}$ Gerrard F Rafferty, ${ }^{2}$ Lalit Kalra ${ }^{4}$}

${ }^{1}$ Faculty of Health, Social Care and Education, St George's, University of London, London, UK ${ }^{2}$ Division of Asthma, Allergy \& Lung Biology, Faculty of Life Sciences and Medicine, King's College London, London, UK
${ }^{3}$ Department of Biostatistics, Institute of Psychiatry, Psychology and Neuroscience, King's College London, London, UK

${ }^{4}$ Department of Basic and Clinical Neuroscience, Institute of Psychiatry, Psychology and Neuroscience, King's College London, London, UK

Correspondence to Dr Stefan T Kulnik, Faculty of Health, Social Care and Education, St George's, University of London, Cranmer Terrace, London SW17 ORE, UK; stefan.kulnik@nhs.net

Acknowledgements Anthony Rudd, David Smithard, Maxine Power, Charles Wolfe and Robin Cant were coapplicants on the study grant. We wish to thank the patients who participated in this study.

Contributors STK: design, data collection, analysis and interpretation, manuscript writing. SSB and GFR: design, data collection, analysis and interpretation. $\mathrm{JH}$ : analysis and interpretation. JM: design, analysis and interpretation. LK: conception and design, data analysis and interpretation. All authors critically revised and approved the final manuscript.

Funding This article presents independent research funded by the UK National Institute for Health Research (NIHR), Research for Patient Benefit Programme (grant reference number PB-PG-0408-16096). The views expressed are those of the authors and not necessarily those of the National Health Service, NIHR or

Department of Health.

Competing interests None declared.

Patient consent Obtained.

Ethics approval UK National Research Ethics Service, South West London Research Ethics Committee 3, reference 10/H0803/32.

Provenance and peer review Not commissioned; externally peer reviewed.

- Additional material is published online only. To view please visit the journal online (http://dx.doi.org/10. 1136/thoraxinl-2015-207810).

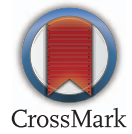

To cite Kulnik ST, Birring SS, Hodsoll J, et al. Thorax 2016;71:474-475.

Received 6 September 2015

Revised 16 December 2015

Accepted 14 January 2016

Published Online First 1 February 2016

Thorax 2016:71:474-475.

doi:10.1136/thoraxjnl-2015-207810

\section{REFERENCES}

1 Widdicombe JG, Addington WR, Fontana GA, et al. Voluntary and reflex cough and the expiration reflex: implications for aspiration after stroke. Pulm Pharmacol Ther 2011;24:312-7.

2 Ward K, Seymour J, Steier J, et al. Acute ischaemic hemispheric stroke is associated with impairment of reflex in addition to voluntary cough. Eur Respir $J$ 2010;36:1383-90.

3 Harraf F, Ward K, Man W, et al. Transcranial magnetic stimulation study of expiratory muscle weakness in acute ischemic stroke. Neurology 2008;71:2000-7.

4 Kulnik S, Rafferty G, Birring $S$, et al. A pilot study of respiratory muscle training to improve cough effectiveness and reduce the incidence of pneumonia in acute stroke: study protocol for a randomized controlled trial. Trials 2014;15:123.

5 Ramsey DJC, Smithard DG, Kalra L. Early assessments of dysphagia and aspiration risk in acute stroke patients. Stroke 2003;34:1252-7.

6 Hannawi Y, Hannawi B, Rao CPV, et al. Stroke-associated pneumonia: major advances and obstacles. Cerebrovasc Dis 2013:35:430-43.

7 Smith CJ, Kishore AK, Vail A, et al. Diagnosis of stroke-associated pneumonia: recommendations from the pneumonia in stroke consensus group. Stroke 2015;46:2335-40. 